\title{
The shelterin complex and hematopoiesis
}

\author{
Morgan Jones, ${ }^{1,2}$ Kamlesh Bisht, ${ }^{3}$ Sharon A. Savage, ${ }^{4}$ Jayakrishnan Nandakumar, ${ }^{3}$ Catherine E. Keegan,, ${ }^{5,6}$ and Ivan Maillard ${ }^{7,8,9}$ \\ 'Graduate Program in Cellular and Molecular Biology, ${ }^{2}$ Medical Scientist Training Program, and ${ }^{3}$ Department of Molecular, Cellular and Developmental Biology, University of Michigan, Ann Arbor, Michigan, \\ USA. ${ }^{4}$ Clinical Genetics Branch, Division of Cancer Epidemiology and Genetics, National Cancer Institute, Bethesda, Maryland, USA. ${ }^{5}$ Department of Human Cenetics, ${ }^{6}$ Department of Pediatrics, ${ }^{7}$ Life Sciences \\ Institute, ${ }^{8}$ Division of Hematology-Oncology, Department of Internal Medicine, and ${ }^{9}$ Department of Cell and Developmental Biology, University of Michigan, Ann Arbor, Michigan, USA.

\begin{abstract}
Mammalian chromosomes terminate in stretches of repetitive telomeric DNA that act as buffers to avoid loss of essential genetic information during end-replication. A multiprotein complex known as shelterin prevents recognition of telomeric sequences as sites of DNA damage. Telomere erosion contributes to human diseases ranging from BM failure to premature aging syndromes and cancer. The role of shelterin telomere protection is less understood. Mutations in genes encoding the shelterin proteins TRF1-interacting nuclear factor 2 (TIN2) and adrenocortical dysplasia homolog (ACD) were identified in dyskeratosis congenita, a syndrome characterized by somatic stem cell dysfunction in multiple organs leading to BM failure and other pleiotropic manifestations. Here, we introduce the biochemical features and in vivo effects of individual shelterin proteins, discuss shelterin functions in hematopoiesis, and review emerging knowledge implicating the shelterin complex in hematological disorders.
\end{abstract}

\section{Organization of the shelterin complex and telomerase at chromosomal ends}

Telomeres, end-replication, and end-protection. Eukaryotic cells contain nucleoprotein complexes known as telomeres that organize and regulate the ends of linear chromosomes. Telomeric DNA consists of repetitive sequences with a short single-stranded, G-rich overhang preceded by a much longer double-stranded region. The average length of telomeric DNA varies between species and tends to decrease in aging cells and tissues. Telomeric DNA is associated with the shelterin complex, a group of six proteins that bind specifically to telomeric DNA: telomere repeat factor 1 (TRF1), TRF2, repressor/activator protein 1 (RAP1), TRF1-interacting nuclear factor 2 (TIN2), protection of telomeres 1 (POT1), and adrenocortical dysplasia homolog (ACD, also referred to as TINT1/PTOP/ PIP1 [TPP1] based on initial descriptions of this protein) (Figure 1 and ref. 1). Shelterin proteins also recruit the telomerase complex to chromosomal ends through an interaction with the telomerase protein component telomerase reverse transcriptase (TERT) (2-7). Together, the telomerase and shelterin complexes solve unique problems caused by linear chromosomes: the end-replication and end-protection problems.

The end-replication problem arises because the DNA replication machinery requires an RNA primer that provides a $3^{\prime}$ hydroxyl group for DNA synthesis at the $5^{\prime}$ end of the lagging strand. Upon primer removal, the resulting gap cannot be filled by replicative DNA polymerases, resulting in DNA loss at chromosomal ends after each replication cycle. Telomerase, a specialized ribonucleoprotein complex, counters this DNA loss by adding telomeric repeats to chromosomal ends and provides a molecular buffer to genetic erosion during end-replication. The telomerase complex contains both the enzymatic activity (encoded by its protein sub-

Conflict of interest: The authors have declared that no conflict of interest exists. Reference information: J Clin Invest. 2016;126(5):1621-1629. doi:10.1172/JCI84547. unit TERT) and the RNA template (present in its RNA subunit telomerase RNA component [TERC]) for reverse transcription. In long-lived proliferative cells, telomere length is maintained by telomerase (8-18).

The end-protection problem is the potential recognition of chromosomal ends as double-stranded breaks (DSBs) by the DNA damage response (DDR) machinery, which could result in deleterious chromosomal end-to-end fusions and genomic instability (Figure 1). Shelterin proteins bind to single- and double-stranded components of telomeric DNA and solve the end-protection problem (1). Without shelterin, telomeres evoke a robust DDR. Exposure of single-stranded G-overhangs elicits an ataxia telangectasia and RAD3-related-mediated (ATR-mediated) DDR, as single-stranded DNA (ssDNA) is bound by replication protein A (RPA) and RPA/ssDNA complexes recruit ATR (19-22). RPA/ ssDNA also interacts with the cell cycle checkpoint complex RAD9/RAD1/HUS1 via RAD17 (23). This complex recruits DNA topoisomerase 2-binding protein 1 (TOPBP1), activating ATR through poorly defined mechanisms (24-26). ATR phosphorylates checkpoint kinase 1 (CHK1) and can trigger cell cycle arrest through regulation of cyclin-dependent kinases or p53 activation, allowing cells the opportunity to repair DNA aberrations (27).

Exposure of the double-stranded portion of telomeres results in aberrant recognition of the telomere as a DSB. DSBs are sensed by the MRE11/RAD50/NBS1 (MRN) complex (28). MRN recruitment triggers ataxia telangectasia mutated (ATM) kinase binding and enhances ATM interaction with CHK2 and p53 (28-30). Activated CHK2 enforces cell cycle arrest directly and through p53 activation $(27,31,32)$. ATM may also activate ATR (33). DDR activation at telomeres primarily activates nonhomologous end joining (NHEJ) and homologous recombination (HR), although additional pathways may be activated (22). Exposure of double-stranded telomeric DNA activates NHEJ, while exposure of ssDNA activates HR $(19,20,34)$. NHEJ-mediated repair at telomeres leads to fusion of different chromosomes or sister 
chromatids, while HR-mediated repair triggers recombination between homologous sister chromatid templates followed by exchange of material between sister chromatids (telomere sister chromatid exchange).

Individual functions of shelterin components as revealed in vitro and in mouse models. The shelterin proteins TRF1 and TRF2 bind double-stranded telomeric DNA; POT1 binds to the single-stranded G-overhang; TIN2 and TPP1 do not bind DNA, but interact with TRF1/2 and POT1, respectively; and RAP1 binds to TRF2 (Figure 1 and ref. 1 ).

Shelterin components play multiple roles in suppressing DDR activation and regulating telomere length. Initial biochemical studies largely used transformed cells and mouse embryonic fibroblasts (MEFs) to elucidate the specific functions of shelterin components. However, while cell culture-based studies provided valuable insights, until recently, the in vivo significance of shelterin dysfunction remained uncharacterized. We now know that individual shelterin proteins play critical roles in vivo in both mice and humans. The unique roles of shelterin proteins in mammalian development and tissue homeostasis are more complex than predictions based on previously reported biochemical functions alone. This profound in vivo significance is most prevalent in tissues that require active turnover and robust stem cell compartments, such as hematopoietic tissues. In addition to developmental defects, several mouse models of shelterin dysfunction revealed features reminiscent of the human syndrome dyskeratosis congenita (35-45).

\section{TRF1/TRF2}

TRF1 and TRF2 play unique roles in telomere protection and length regulation (46-49). TRF1 overexpression results in telomere shortening, while dominant-negative TRF1 leads to telomere lengthening (50). Though TRF1 and TRF2 are not known to directly interact with telomerase, they facilitate formation of higher-order telomeric structures (51). TRF1 isoforms have also been linked to cell cycle control (52). In MEFs, Trf1 inactivation results in widespread stalling of the DNA replication machinery at telomeres and the formation of gaps, known as fragile telomeres $(53,54)$. Trf1 inactivation and subsequent replicative dysfunction induces robust DDR activation involving ATM, ATR, p53, and retinoblastoma $(\mathrm{RB})$, leading to cellular senescence $(53,54)$. Trfi is essential for embryonic development, as germline Trf1 inactivation results in lethality before E6.5 (55). While $p 53$ inactivation rescued MEFs from the effects of Trf1 deletion, a similar rescue was not observed in embryos, suggesting that differences exist between the effects of shelterin deficiencies in cell culture as compared with the entire organism.

$\operatorname{Trf1}$ inactivation in the $\mathrm{BM}$ results in hematopoietic failure within weeks (45). $\operatorname{Trf1^{-/-}}$ hematopoietic stem cells (HSCs) had impaired repopulation potential in transplantation assays that was linked to p53 activation in the absence of a significant apoptotic response. Instead, $p 21$-mediated senescence with associated telomere shortening was documented. In addition, $\operatorname{Trf1}$ inactivation in the skin resulted in severe epidermal defects and neonatal mortality due to loss of skin barrier function (54). Interestingly, p53 inactivation in the skin was sufficient to prevent lethality. However, this rescue strategy resulted in squamous cell carcinomas, demon- strating that genomic instability due to $\operatorname{Trf1}$ loss is tumorigenic. The ability of $p 53$ inactivation to rescue developmental abnormalities in the skin, but not in the entire embryo, suggests that defined cell types and tissues may differentially activate DDR signaling in response to shelterin defects.

TRF2 is structurally related to TRF1 and specifically prevents ATM activation at telomeric ends $(19,20,56,57)$. Trf 2 inactivation results in NHEJ-driven telomeric fusion events independent of replication fork stalling $(34,57-59)$. TRF2 helps form higher-order telomeric structures known as t-loops, which have been proposed to be instrumental for TRF2's functions $(51,60,61)$. TRF2 overexpression in telomerase-positive human cells leads to telomere shortening, while telomere elongation is observed when TRF2 levels decrease (51). As with TRF1, this finding is not related to regulation of telomerase expression or activity (51).

In mice, constitutive $\operatorname{Tr} 2$ deficiency led to embryonic lethality that was not rescued by $p 53$ deficiency (56). $\operatorname{Tr} f 2$ overexpression in the skin resulted in hyperpigmentation and predisposed mice to squamous cell carcinomas (41). Epithelial cells from mice with $\operatorname{Trf} 2$ overexpression had prominent telomere shortening that was not related to telomerase dysfunction. The combination of telomerase deficiency and $\operatorname{Trf} 2$ overexpression led to accelerated tumorigenesis as the result of enhanced telomere dysfunction and fusion events (40).

\section{RAP1}

RAP1 (encoded by the gene TERF2IP) is localized at telomeres via its interaction with TRF2 $(62,63)$. Terf2ip is the only shelterin gene whose inactivation does not cause embryonic lethality in mice, suggesting no essential function in end-protection or replication (37). Initial studies indicated that RAP1 was involved in preventing NHEJ at telomeres, while later work suggested a role in preventing HR $(37,62,64-67)$. Recently, RAP1 was also found to perform important extratelomeric functions that provide protection against obesity in mice $(68,69)$. In addition, Terf2ip gene inactivation results in hyperpigmentation, telomere shortening, and increased DDR activation in adult mice (37). Together, these findings suggest that RAP1 plays an important role in telomere homeostasis. Studies of Terf 2 ip illustrate the concept that in vivo observations are required to advance our understanding of shelterin protein functions.

\section{POT1}

POT1 binds the single-stranded G-overhang of telomeric DNA, which requires stable heterodimer formation with TPP1 in vivo (19, 70-76). POT1 is required for suppression of ATR-mediated DDR and for regulation of $3^{\prime}$ G-strand overhang length (77). In mice, this function is achieved through complex regulation of $5^{\prime}$ C-strand resection and subsequent fill-in (78). The mouse Pot1 gene underwent duplication, creating Pot1a and Pot1b (79). Pot1a is critical for ATR inhibition, while Pot1b is involved in telomere length regulation and prevents excessive $5^{\prime}$ resection $(42,43,79$, 80). Pot1a loss results in p53-dependent senescence induction (80). In the presence of telomerase, only combined Pot $1 a / b$ loss leads to increased telomeric instability $(77,79)$. Furthermore, Pot1 $a / b$ loss causes endoreduplication, resulting in MEFs with $>4 \mathrm{~N}$ DNA (79). However, combined Pot1b deficiency and telomerase 


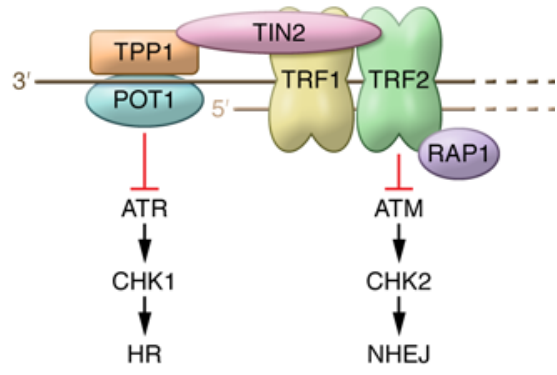

Figure 1. Organization and molecular functions of the shelterin complex at telomeric ends. Telomeric DNA contains repetitive sequences forming a long double-stranded region (right) followed by a shorter G-rich, singlestranded overhang (left). The shelterin complex consists of six proteins (TRF1, TRF2, RAP1, TIN2, POT1, and TPP1) that bind the double-stranded and single-stranded regions of telomeric DNA. TPP1 is encoded by the gene $A C D$. Shelterin proteins play important, nonredundant roles in preventing activation of DDR pathways at chromosome ends, including ATR/CHK1 activation by exposed ssDNA and ATM/CHK2 activation by doublestranded DNA. Shelterin proteins prevent attempted repair by HR and NHEJ mechanisms. Additional pathways and crosstalk between pathways exist but are not represented here for the sake of simplicity.

haploinsufficiency result in telomere shortening and increased chromosomal fusions $(42,43)$. By virtue of binding tightly to the $3^{\prime}$ overhang at chromosome ends, POT1 serves as a natural inhibitor of telomerase. Indeed, disruption of POT1's DNA binding domain results in excessive telomere elongation, suggesting unregulated access of telomerase to chromosome ends (81).

Pot1a deletion causes early embryonic lethality, while Pot1bdeficient mice are born in normal Mendelian ratios $(43,79,80)$. This suggests a critical role for Pot1a but not Pot1b during embryonic development. Despite the normal development of Pot1b-deficient mice, males eventually became infertile with reduced sperm production. Subsequent genetic models in which Pot1b deficiency was combined with Terc haploinsufficiency resulted in the first in vivo models with a phenotype reminiscent of dyskeratosis congenita as a result of shelterin dysfunction $(42,43)$. In contrast, complete loss of telomerase activity in the setting of Pot1b deficiency resulted in embryonic lethality, perhaps due to excessive chromosomal shortening during early development. Mice deficient for Pot1b with combined Terc haploinsufficiency demonstrated a spectrum of phenotypes reminiscent of dyskeratosis congenita patients, including skin hyperpigmentation, testicular atrophy, increased apoptosis in intestinal crypts, and reduced lifespan in the context of telomere shortening. Reduced life expectancy was attributed to the development of hematopoietic failure. Mechanistically, Pot $1 b^{-/-\mathrm{TerC}^{+} /-}$HSCs were reduced in number and failed to compete with WT HSCs in transplantation assays (44). Hematopoietic progenitor cells had increased p53 activation with a bias toward apoptosis rather than cell cycle arrest. p53 loss markedly rescued HSC function, demonstrating that $\mathrm{p} 53$ plays a critical role

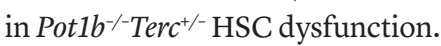

\section{ACD/TPP1}

In vivo, POT1 binding requires TPP1, the protein product of the $A C D$ gene $(19,36,73-76,82)$. Distinct functions of TPP1 can be traced to biochemical functions of TPP1's protein domains, which form binding interfaces with specific partners: POT1, TIN2, and TERT. With these characteristics, TPP1 functions as a molecular hub coordinating end-protection and end-replication functions of the shelterin/telomerase complex.

Initial studies in $\mathrm{Acd}^{-/}$MEFs revealed that many consequences of $A c d$ deficiency phenocopied Pot1a/b inactivation. These studies indicated that the suppression of ATR signaling and HR through POT1 is the central function of TPP1 (74). Additional studies implicated TPP1 in preventing ATM activation and suppressing NHEJ, suggesting a more complicated role in end-protection $(19,83,84)$. MEF-based studies showed that most effects of Acd inactivation in cultured cells are driven by p53-mediated growth arrest $(19,84)$. However, subsequent work suggested that p53-independent effects of Acd loss exist, as MEFs immortalized with SV40 large $\mathrm{T}$ antigen (which inactivates p53 and RB) still demonstrate growth arrest upon Acd loss (74).

Apart from its end-protection function, TPP1 also plays complex roles in regulating telomerase activity. Work in human cell lines demonstrated that TPP1 is required for recruitment of both telomerase and POT1 $(2,3,73)$. While POT1 recruitment is expected to negatively regulate telomere lengthening, recruitment of telomerase by TPP1 would result in telomere lengthening. Indeed, the telomere length phenotype of TPP1 knockdown is variable and context dependent. While TPP1 knockdown in cultured cancer cell lines resulted in telomere lengthening, telomere shortening was observed in skin keratinocytes from newborn mice with keratinocyte-specific deletion of $\mathrm{Acd}\left(\mathrm{K}^{2}-\mathrm{Cre}^{+} \mathrm{Acd} \mathrm{d}^{\mathrm{f} / \mathrm{f})}\right.$ (76, 84). How TPP1 coordinates its contrasting telomerase-regulating activities remains to be determined.

The telomerase recruitment activity of TPP1 was attributed to the oligonucleotide/oligosaccharide binding $(\mathrm{OB})$ fold domain of TPP1. Recent studies identified a small region in the TPP1 OB fold (the TEL patch) that is both necessary and sufficient for telomerase recruitment (Figure 2 and refs. 4-6). Thus, TPP1 is not required solely for DDR suppression but also for telomere elongation, suggesting the need to study TPP1 function in telomeraseexpressing cells, including stem cells and cancer cells. In addition to telomerase recruitment, TPP1 promotes telomerase processivity in the presence of POT1 (85). How POT1, a negative regulator of telomerase, teams up with TPP1 to stimulate telomerase processivity is not fully understood, although models based on telomerase enzyme kinetics have been proposed (86).

A recent study showed that TPP1 interacts with another ssDNA-binding complex known as the CST complex (CTC1, STN1, and TEN1) to limit excessive telomere elongation (87). At early steps of telomere replication, TPP1 recruits telomerase and promotes telomere extension. As replication progresses, CST binds the elongating $\mathrm{G}$-strand, preventing further association with telomerase. Additionally, the presence of CST inhibits TPP1's ability to enhance telomerase processivity.

In mice, complete Acd inactivation leads to embryonic lethality (74). However, a spontaneously occurring splice variant resulting in a hypomorphic allele (acd) revealed essential developmental functions for Acd (36). Depending on the genetic background, mice homozygous for hypomorphic acd displayed adrenocortical dysplasia (giving the gene its original name), caudal truncation, genitourinary abnormalities, infertility, skin 


\section{A TPP1}

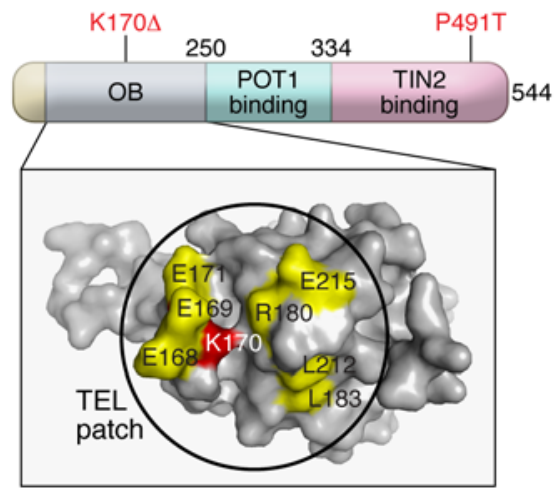

B TIN2

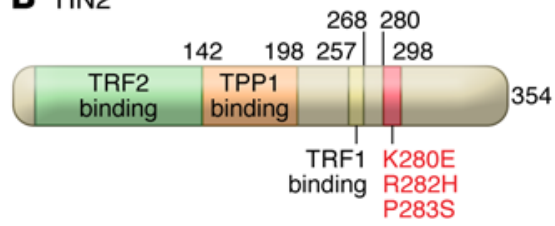

Figure 2. Structure of TPP1 and TIN2 shelterin proteins, highlighting key mutations identified in patients with dyskeratosis congenita. (A) TPP1 (encoded by $A C D$ ) contains an OB fold domain (OB), which recruits telomerase through an exposed TEL patch, as well as a POT1-binding domain and a TIN2-binding domain. Human variants identified in dyskeratosis congenita families are shown in red (K170 $\Delta, \mathrm{P} 491 \mathrm{~T})$. Essential surface-exposed amino acids in the TEL patch are shown in yellow, while the mutation identified in dyskeratosis congenita is shown in red. Numbers indicate amino acid positions. (B) TIN2 (encoded by TINF2) contains an $\mathrm{N}$-terminal TRF2-binding region, followed by TPP1-binding and TRF1-binding domains. Selected recurrent mutations identified in dyskeratosis congenita patients are indicated in red (K280E, R282H, P283S). Multiple other mutations affecting the 280-300 amino acid cluster were reported but are not depicted. Note that the hotspot for dyskeratosis congenita mutations lies outside of all known protein-binding regions of TIN2. Numbers indicate amino acid positions.

hyperpigmentation, and strain-dependent prenatal or perinatal lethality. A subsequent study showed that $A c d$ inactivation in the skin caused hyperpigmentation, epidermal stem cell defects, and neonatal mortality due to loss of barrier function (84). With both skin-specific Acd deletion and $a c d$-homozygous mice, most phenotypic abnormalities could be rescued by $p 53$ inactivation $(35,84,88)$. Interestingly, $p 53$ inactivation failed to rescue strain-specific embryonic lethality in acd homozygotes (35). These data reveal critical tissue-specific and developmental functions for Acd and suggest that p53-dependent and p53-independent mechanisms underlie these effects.

We recently demonstrated that Acd critically regulates HSC homeostasis (39). In mice with hypomorphic acd alleles, we observed G2/M arrest in fetal hematopoietic progenitors. These cells could not provide hematopoietic reconstitution in irradiated recipients, demonstrating marked HSC dysfunction. When Acd was inactivated using a conditional allele, HSCs were rapidly depleted within less than one week in adult mice, and these cells were unable to provide stable engraftment after competitive or noncompetitive transplantation. In addition to $\mathrm{G} 2 / \mathrm{M}$ arrest, hematopoietic progenitors demonstrated chromosomal instabil- ity, as indicated by fusion events and robust caspase activation within 48 hours of $A c d$ deletion. Interestingly, p53 deletion did not rescue $A c d$-deficient HSCs, even transiently. These findings contrast with the rescue of Acd-deficient epidermal stem cells by p53 inactivation, suggesting that distinct stem cell populations respond differently to $A c d$ loss. Moreover, the functional rescue of Pot $1 b$-deficient HSCs by $p 53$ inactivation suggests that Acd's effects in HSCs are not solely due to POT1B destabilization (44, 84). Thus, the relationship between shelterin deficiency and p53 activation is more complicated than previously anticipated.

\section{TIN2}

TIN2 is an adaptor protein that plays critical roles in stabilizing the shelterin complex. TIN2 binds TRF1 and TRF2, stabilizing TRF1 and TRF2 at telomeres (89). TIN2 also binds TPP1 and is required for TPP1/POT1's recruitment to the shelterin complex (90). TIN2 loss results in ATR activation and excessive $3^{\prime}$ overhang generation (from TPP1/POT1 loss) and ATM activation (from TRF2 destabilization). These data highlight the critical function of TIN2 in organizing the shelterin complex (38). TIN2 knockdown also results in decreased telomerase recruitment to telomeres, most likely because TIN2 is required for telomeric recruitment of TPP1 (3). TIN2 may have additional roles outside of telomere homeostasis. TIN2 was detected in the mitochondria in a human cancer cell line, and TIN2 knockdown led to abnormal mitochondrial morphology (91). TIN2 loss promoted increased oxidative metabolism and reduced glycolysis in human cancer cells. These data indicate that TIN2 may have telomeric and extratelomeric functions, and they suggest the possibility that other shelterin components may also function away from telomeres.

Tin2 loss causes embryonic lethality in mice (92). Conditional Tin2 inactivation has not been characterized in vivo. However, TINF2 mutations were identified in human dyskeratosis congenita patients, providing an impetus for in vivo modeling of TIN2 function (93-97). To this end, a knock-in approach in which a Tin2 allele with lysine 267 replaced with glutamate (K267E, synonymous with the dyskeratosis congenita-causing K280E mutation in humans) was utilized (Figure 2 and ref. 38). Homozygous mice were not viable, but heterozygous mice demonstrated reductions in neutrophil, reticulocyte, and platelet counts that worsened with increasing generations. This type of disease anticipation is observed in mice or patients with telomerase defects, as they inherit shortened telomeres through the germline. In addition to the hematopoietic phenotype, heterozygous mice were smaller than WT littermates and born in reduced numbers, with worsening of the phenotype in later generations. Male mice harboring a single mutant allele had reduced fertility. This model also revealed apparent telomerase-independent telomere shortening. Mice harboring the Tin2-K267E allele had shorter telomeres than WT littermates. When this allele was crossed onto a Terc-deficient background, telomeres shortened to a greater extent than in mice null for Terc alone. Given that the hotspot for dyskeratosis congenita mutations in TINF2 encodes a region of the protein that is dispensable for TRF1, TRF2, and TPP1 binding, it is unclear how these mutations result in telomere shortening. More work is needed to understand the molecular and cellular consequences of TINF 2 mutations. 


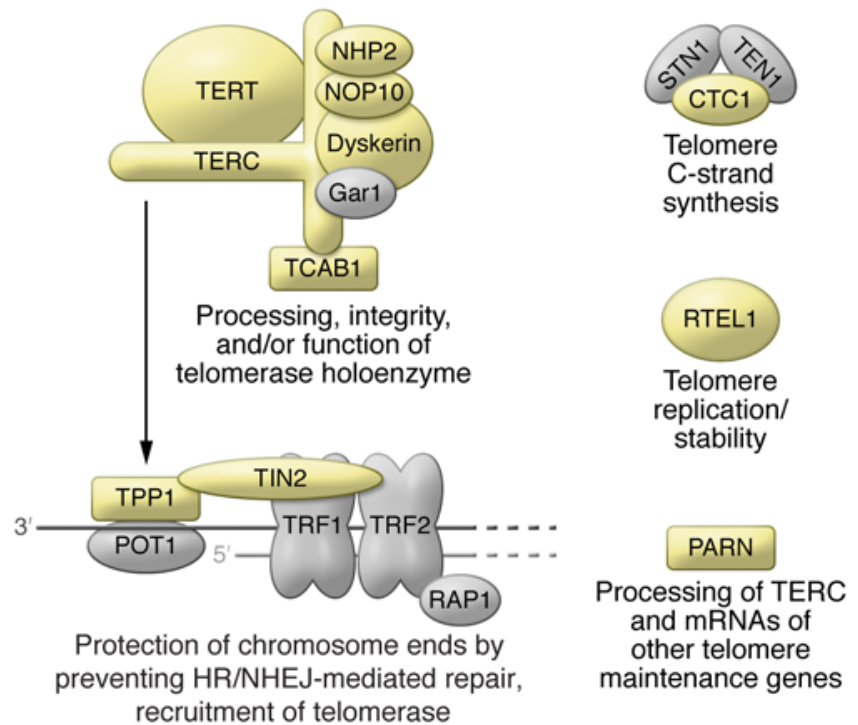

Figure 3. Schematic representation of human proteins affected by germline mutations in dyskeratosis congenita and related disorders. Mutations affecting 11 different proteins have been reported to date in dyskeratosis congenita and related disorders, as indicated by colored structures. TERT, TERC, dyskerin, NHP2, NOP10, and TCAB1 are important for the processing, integrity, and/ or function of the telomerase holoenzyme, a ribonucleoprotein complex containing TERC RNA and the catalytic TERT protein with reverse transcriptase activity. TIN2 and TPP1 (encoded by ACD) are members of the shelterin complex (see Figure 1). CTC1 belongs to a complex that regulates telomere C-strand synthesis and telomere replication. RTEL1 is important for telomere replication and stability. PARN was recently described to control processing of TERC RNA as well as mRNAs of other telomere maintenance genes.

\section{Shelterin and human BM failure syndromes}

Recent studies in dyskeratosis congenita highlight the importance of the shelterin complex in hematopoiesis. Dyskeratosis congenita is a human telomere biology disorder with pleiotropic manifestations that frequently causes BM failure $(98,99)$. Hematopoietic stem and progenitor cells from patients with dyskeratosis congenita have defective self-renewal, suggesting that these hematopoietic cell-intrinsic defects are the primary cause of hematopoietic failure in these patients. In addition, HSC niche defects could also contribute to the phenotype (100).

Dyskeratosis congenita was first linked to mutations in DKC1 - which encodes dyskerin, an accessory protein required for telomerase stability and function - and then in genes encoding components of telomerase itself: TERC, encoding the telomerase RNA subunit, and TERT, encoding the telomerase enzymatic protein subunit (Figure 3 and refs. 101-104). Other mutations were described in NOP1O (encoding a small nucleolar ribonucleoprotein), NHP2 (encoding a small nucleolar ribonucleoprotein subunit), and WRAP53 (encoding TCAB1), which affect telomerase trafficking, assembly, or stability (105-107); in RTEL1 (encoding an RNA helicase that regulates telomere unwinding and replication) (108-112); in CTC1 (encoding a component of the CST complex) (113-115); and in PARN (encoding a poly[A]-specific ribonuclease) $(116,117)$. In addition, mutations in two genes encoding elements of the shelterin complex (TINF2 and ACD) were identified in families with dyskeratosis congenita and dyskeratosis congenita-related disorders, as discussed in more detail below $(93,94,118,119)$. Altogether, mutations in a total of 11 genes have been reported to date, including X-linked, autosomal dominant, and autosomal recessive inheritance patterns.

Dyskeratosis congenita patients are classically diagnosed by the "mucocutaneous triad" of oral leukoplakia, abnormal skin pigmentation, and nail dystrophy. However, the clinical manifestations are pleiotropic, and penetrance is variable; thus, the classic triad is not present in all patients. Up to $85 \%$ of dyskeratosis congenita patients eventually develop hematopoietic failure, establishing dyskeratosis congenita as an inherited BM failure syndrome $(99,102,120)$. Dyskeratosis congenita patients are also at risk of liver disease and pulmonary fibrosis, suggesting that somatic stem/progenitor cell populations in these organs are susceptible to telomere dysregulation (121). Additionally, dyskeratosis congenita is a cancer predisposition syndrome, as approximately $40 \%$ of patients with dyskeratosis congenita develop cancer by the age of 50, with myelodysplastic syndromes, acute myeloid leukemia, and squamous cell carcinoma being most common (102). Up to $70 \%$ of dyskeratosis congenita cases have an identifiable mutation in one of the above genes, while the remaining $30 \%$ remain of unknown genetic origin (102). Thus, our knowledge of the genetic causes of dyskeratosis congenita remains incomplete, and additional contributing genes must be considered.

\section{TINF2}

The first shelterin gene implicated in dyskeratosis congenita was TINF2 $(93,94)$. Patients with TINF2 mutations usually have shorter telomeres with symptom onset at an earlier age than patients with TERC or TERT mutations $(93,96,122,123)$. TINF2 mutations are associated with Hoyeraal-Hreidarsson syndrome (a dyskeratosis congenita variant with cerebellar hypoplasia and immunodeficiency) and Revesz syndrome (with bilateral exudative retinopathy) $(93,94,124,125)$. To date, approximately 20 mutations have been identified in TINF 2 in patients, affecting a recurrent hotspot in the gene (Figure 2 and ref. 125). TINF2 mutations act in an autosomal dominant fashion but often appeared to arise de novo in dyskeratosis congenita patients (93). At least two alternative mechanisms of telomere dysfunction have been proposed for TINF2 mutations. Biochemical analysis of a subset of disease-causing TINF2 mutations showed reduced telomerase recruitment to telomeres in a TPP1-dependent fashion (126). Another study using a knockin approach reported decreased telomerase recruitment without changes in TPP1 localization to telomeres (127). These data suggest a mechanism for progressive telomere shortening due to defective telomerase recruitment, although they fail to explain the early age of dyskeratosis congenita onset, increased clinical complications, or exceedingly short telomeres in the setting of TINF2 mutations as compared with other dyskeratosis congenita etiologies. Another study showed that dyskeratosis congenita-associated mutations in TIN2 interfere with binding of TIN2 to heterochromatin protein $1 \gamma(\mathrm{HP} 1 \gamma)$, which results in defective sister telomere cohesion in cultured human cells (128). Recent work focusing on the K280E mutation, one of the initially characterized disease-causing TINF2 mutations, suggests that at least part of the disease phenotype can be mediated by telomerase-independent telomere shortening and DDR activation $(38,94)$. 
Table 1. Germline and somatic shelterin gene mutations reported in human cancer

\begin{tabular}{|c|c|c|c|c|}
\hline Gene & Mutation & Type of cancer & Nature & References \\
\hline \multirow[t]{4}{*}{ TERF2IP } & M5I & Familial Melanoma & Germline & 135 \\
\hline & $\mathrm{D} 10 \mathrm{H}$ & & & \\
\hline & Q191R & & & \\
\hline & R364X & & & \\
\hline \multirow[t]{6}{*}{$A C D$} & A200T & Familial Melanoma & Germline & 135 \\
\hline & N249S & & & \\
\hline & V272M & & & \\
\hline & Q320X & & & \\
\hline & I322F & & & \\
\hline & G223V & $\begin{array}{c}\text { Acute Lymphoblastic } \\
\text { Leukemia }\end{array}$ & Somatic & 138 \\
\hline \multirow[t]{22}{*}{ POT1 } & M1L & CLL & Somatic & 132,133 \\
\hline & Y36N & & & \\
\hline & Y66X & & & \\
\hline & K90Q & & & \\
\hline & Q94R & & & \\
\hline & Y223C & & & \\
\hline & S250X & & & \\
\hline & $\mathrm{H} 266 \mathrm{~L}$ & & & \\
\hline & G272V & & & \\
\hline & C591W & & & \\
\hline & Y89C & Familial melanoma & Germline & 136 \\
\hline & Q94E & & & \\
\hline & R273L & & & \\
\hline & R137H & $\begin{array}{l}\text { Cutaneous Malignant } \\
\text { Melanoma }\end{array}$ & Germline & 137 \\
\hline & $\mathrm{D} 224 \mathrm{~N}$ & & & \\
\hline & S270N & & & \\
\hline & A532P & & & \\
\hline & Q623H & & & \\
\hline & C95C & Familial Clioma & Germline & 139 \\
\hline & E450X & & & \\
\hline & D617E & & & \\
\hline & R117C & Cardiac Angiosarcoma & Germline & 140 \\
\hline
\end{tabular}

\section{ACD}

Two recent reports identified germline $A C D$ mutations in families with inherited $\mathrm{BM}$ failure and dyskeratosis congenita spectrum disorders (Figure 2 and refs. 118, 119). One proband presented with features of dyskeratosis congenita by the age of 10 months and blood cytopenias by 20 months, requiring matched unrelated allogeneic hematopoietic cell transplantation by 3 years of age (118). This patient was ultimately diagnosed with Hoyeraal-Hreidarsson syndrome due to concomitant cerebellar hypoplasia and dyskeratosis congenita features. In this study, an ACD mutation causing in-frame loss of lysine 170 in TPP1 was present in the proband and other family members with very short telomeres. This mutation resulted in a structural change in the TEL patch that abrogated TPP1's ability to bind telomerase, with functional consequences similar to those of previously reported experimental mutations affecting the TEL patch (4-6). Interestingly, the proband in this study also carried a mutation substituting threonine for proline at position 491 in the TIN2-interacting domain of TPP1. While this mutation did not affect telomerase recruitment, it resulted in modest disruption of the TPP1/TIN2 interaction, the significance of which remains unknown. Another study characterized a family in which several generations were affected by aplastic anemia (119). In this family, the proband presented at age 8 with worsening pancytopenia. Despite being unrelated to the family in the other study, affected individuals had the same deletion of lysine 170 in TPP1. These independent observations highlight the critical role of TPP1's TEL patch region in telomere homeostasis. The variability in disease presentation in these two studies was consistent with dyskeratosis congenita caused by mutations in other genes, including TERT and RTEL1, where autosomal dominant and recessive inheritance patterns have been reported.

In individuals with TEL patch mutations, defective recruitment of telomerase to telomeric ends is predicted to result in telomere shortening, and indeed very short telomeres were observed in affected individuals $(118,119)$. These conclusions were supported by the study of engineered mutations in embryonic stem cells (129). However, TEL patch mutations have not been modeled in vivo, and the suggested pathogenic mechanisms remain to be fully investigated. In particular, we do not know if human shelterin gene mutations exert all their effects via their impact on telomere length or if other mechanisms such as end-deprotection are involved. Development of mouse models to characterize how focal shelterin defects affect telomere homeostasis could provide fundamental insights into dyskeratosis congenita pathogenesis and the role of shelterin proteins in tissue aging.

\section{Shelterin and hematological malignancies}

Poncet and colleagues reported alterations in the expression pattern of several shelterin genes in chronic lymphocytic leukemia (CLL) (130). Expression of TRF1, RAP1, and POT1 was reduced, while $A C D$ expression was higher than in normal lymphocytes. Subsequent work sought to determine if shelterin dysfunction could play a pathogenic role in CLL. Patients with early-stage CLL had an increased frequency of dysfunctional telomeres, as indicated by the localization of DDR-associated proteins at telomeric ends (telomere dysfunction-induced foci, TIFs) (131). TIFs were identifiable before telomere shortening could be observed. After surveying shelterin genes and genes associated with telomeric maintenance, it was found that expression of TINF2 and ACD was reduced. Thus, telomere deprotection may take place in CLL in addition to subsequent telomere erosion, although the significance of these findings remains to be fully explored.

Further supporting the idea that telomere deprotection may play a role in CLL, POT1 point mutations were reported in 5\% of CLL cases $(132,133)$. Most of these mutations were in regions encoding the two OB fold-containing domain of POT1, which may hinder POT1's ability to bind telomeric DNA. Patients harboring these mutations had increased frequencies of sister chromatid fusions, chromosome fusions, and multitelomeric signals or fragile telomeres (a feature associated with replication fork stalling at telomeric ends). It is interesting to speculate whether CLL has unique characteristics that make it particularly sensitive to shelterin dysfunction as compared with other cancers. Although telomere erosion and genomic instability have been documented in CLL (134), similar investi- 
gations have not yet been performed in detail in other hematological malignancies. Of note, mutations in the shelterin genes POT1, $A C D$, and TERF2IP have been described in familial melanoma and other cancers, indicating a broad potential relevance in oncogenesis (135-137). Table 1 summarizes all the germline or somatic mutations in shelterin genes described to date in human cancer, although their mechanisms of action and significance remain to be fully explored.

\section{Conclusions and perspectives}

With the identification of $A C D$ and TINF2 mutations in dyskeratosis congenita patients, two out of six shelterin genes have now been associated with human hematopoietic failure syndromes (Figure 3). At least $30 \%$ of patients presenting with dyskeratosis congenita or dyskeratosis congenita-like syndromes still have no known genetic defects; thus, it is possible that additional shelterin gene mutations will be discovered in these disorders. In addition, shelterin dysfunction or mutations may underlie other human diseases, including developmental defects and cancer. Much progress has been made in understanding the complex biology of telomere homeostasis, particularly in terms of how end-elongation and end-protection are coordinately regulated. In the future, targeted genetic interventions could be particularly informative in studying the function of individual shelterin proteins, as global loss or downregulated expression of shelterin components can trigger widespread defects that mask their precise functions. For example, engineering human mutations and polymorphisms - as well as point mutations targeting specific protein-protein interfaces - could reveal important, clinically relevant phenotypes. In vivo studies will be necessary to fully understand the molecular functions of shelterin proteins in individual tissues, including in the regulation of hematopoietic homeostasis.

\section{Acknowledgments}

Experimental work in the Nandakumar, Keegan, and Maillard laboratories has been supported by the NIH: R01-AG-050509 (to J. Nandakumar, C.E. Keegan, and I. Maillard); R00-CA-167644 and P30-AG-013283 (to J. Nandakumar); R01-HD-058606 (to C.E. Keegan); and R01-AI-091627 (to I. Maillard). M. Jones was supported by T32-GM007315 and T32-GM007863. I. Maillard is the recipient of a career development award from the Leukemia and Lymphoma Society (grant 1227-14) and an award from the D. Dan and Betty Kahn Foundation. Research in the Savage laboratory has been supported by the intramural research program of the Division of Cancer Epidemiology and Genetics, National Cancer Institute of the NIH.

Address correspondence to: Ivan Maillard, Life Sciences Institute \#6382A, 210 Washtenaw Avenue, University of Michigan, Ann Arbor, Michigan 48109, USA. Phone: 734.763.3599; E-mail: imaillar@umich.edu.
1. Palm W, de Lange T. How shelterin protects mammalian telomeres. Annu Rev Genet. 2008;42:301-334.

2. Xin H, et al. TPP1 is a homologue of ciliate TEBP- $\beta$ and interacts with POT1 to recruit telomerase. Nature. 2007;445(7127):559-562.

3. Abreu E, et al. TIN2-tethered TPP1 recruits human telomerase to telomeres in vivo. Mol Cell Biol. 2010;30(12):2971-2982.

4. Zhong FL, Batista LF, Freund A, Pech MF, Venteicher AS, Artandi SE. TPP1 OB-fold domain controls telomere maintenance by recruiting telomerase to chromosome ends. Cell. 2012;150(3):481-494.

5. Nandakumar J, Bell CF, Weidenfeld I, Zaug AJ, Leinwand LA, Cech TR. The TEL patch of telomere protein TPP1 mediates telomerase recruitment and processivity. Nature. 2012;492(7428):285-289.

6. Sexton AN, Youmans DT, Collins K. Specificity requirements for human telomere protein interaction with telomerase holoenzyme. J Biol Chem. 2012;287(41):34455-34464.

7. Schmidt JC, Cech TR. Human telomerase: biogenesis, trafficking, recruitment, and activation. Genes Dev. 2015;29(11):1095-1105.

8. Greider CW, Blackburn EH. Identification of a specific telomere terminal transferase activity in Tetrahymena extracts. Cell. 1985;43(2 pt 1):405-413.

9. Greider CW, Blackburn EH. A telomeric sequence in the RNA of Tetrahymena telomerase required for telomere repeat synthesis. Nature. 1989;337(6205):331-337.

10. Lingner J, Hughes TR, Shevchenko A, Mann M, Lundblad V, Cech TR. Reverse transcriptase motifs in the catalytic subunit of telomerase. Science.
1997;276(5312):561-567.

11. Lee HW, et al. Essential role of mouse telomerase in highly proliferative organs. Nature. 1998;392(6676):569-574.

12. Rudolph KL, et al. Longevity, stress response, and cancer in aging telomerase-deficient mice. Cell. 1999;96(5):701-712.

13. Blasco MA, et al. Telomere shortening and tumor formation by mouse cells lacking telomerase RNA. Cell. 1997;91(1):25-34.

14. Greenberg RA, Allsopp RC, Chin L, Morin GB, DePinho RA. Expression of mouse telomerase reverse transcriptase during development, differentiation and proliferation. Oncogene. 1998;16(13):1723-1730.

15. Allsopp RC, Morin GB, DePinho R, Harley CB, Weissman IL. Telomerase is required to slow telomere shortening and extend replicative lifespan of HSCs during serial transplantation. Blood. 2003;102(2):517-520.

16. Wright WE, Piatyszek MA, Rainey WE, Byrd W, Shay JW. Telomerase activity in human germline and embryonic tissues and cells. Dev Genet. 1996;18(2):173-179.

17. Morrison SJ, Prowse KR, Ho P, Weissman IL. Telomerase activity in hematopoietic cells is associated with self- renewal potential. Immunity. 1996;5(3):207-216.

18. Prowse KR, Greider CW. Developmental and tissue-specific regulation of mouse telomerase and telomere length. Proc Natl Acad Sci US A. 1995;92(11):4818-4822.

19. Guo X, et al. Dysfunctional telomeres activate an ATM-ATR-dependent DNA damage response to suppress tumorigenesis. EMBO J. 2007;26(22):4709-4719.

20. Denchi EL, de Lange T. Protection of telo- meres through independent control of ATM and ATR by TRF2 and POT1. Nature. 2007;448(7157):1068-1071.

21. Zou L, Elledge SJ. Sensing DNA damage through ATRIP recognition of RPA-ssDNA complexes. Science. 2003;300(5625):1542-1548.

22. Sfeir A, de Lange T. Removal of shelterin reveals the telomere end-protection problem. Science. 2012;336(6081):593-597.

23. Zou L, Liu D, Elledge SJ. Replication protein A-mediated recruitment and activation of Rad17 complexes. Proc Natl Acad Sci US A. 2003;100(24):13827-13832.

24. Lee J, Kumagai A, Dunphy WG. The Rad9Hus1-Rad1 checkpoint clamp regulates interaction of TopBP1 with ATR. J Biol Chem. 2007;282(38):28036-28044.

25. Kumagai A, Lee J, Yoo HY, Dunphy WG. TopBP1 activates the ATR-ATRIP complex. Cell. 2006;124(5):943-955.

26. Mordes DA, Glick GG, Zhao R, Cortez D. TopBP1 activates ATR through ATRIP and a PIKK regulatory domain. Genes Dev. 2008;22(11):1478-1489.

27. Smith J, Tho LM, Xu N, Gillespie DA. The ATMChk2 and ATR-Chk1 pathways in DNA damage signaling and cancer. Adv Cancer Res. 2010;108.

28. Lee JH, Paull TT. ATM activation by DNA double-strand breaks through the Mre11-Rad50Nbs1 complex. Science. 2005;308(5721):551-554.

29. Lee JH, Paull TT. Direct activation of the ATM protein kinase by the Mre11/Rad50/Nbs1 complex. Science. 2004;304(5667):93-96.

30. Uziel T, Lerenthal Y, Moyal L, Andegeko Y, Mittelman L, Shiloh Y. Requirement of the MRN complex for ATM activation by DNA damage. EMBO J. 2003;22(20):5612-5621.

31. Shechter D, Costanzo V, Gautier J. Regulation of 
DNA replication by ATR: signaling in response to DNA intermediates. DNA Repair (Amst). 2004;3(8-9):901-908.

32. Shiloh Y. ATM and ATR: networking cellular responses to DNA damage. Curr Opin Genet Dev. 2001;11(1):71-77.

33. Jazayeri A, et al. ATM- and cell cycle-dependent regulation of ATR in response to DNA double-strand breaks. Nat Cell Biol. 2006;8(1):37-45.

34. Rai R, et al. The function of classical and alternative non-homologous end-joining pathways in the fusion of dysfunctional telomeres. $Е M B O \mathrm{~J}$. 2010;29(15):2598-2610.

35. Vlangos CN, O'Connor BC, Morley MJ, Krause AS, Osawa GA, Keegan CE. Caudal regression in adrenocortical dysplasia (acd) mice is caused by telomere dysfunction with subsequent p53-dependent apoptosis. Dev Biol. 2009;334(2):418-428.

36. Keegan CE, et al. Urogenital and caudal dysgenesis in adrenocortical dysplasia (acd) mice is caused by a splicing mutation in a novel telomeric regulator. Hum Mol Genet. 2005;14(1):113-123.

37. Martinez P, et al. Mammalian Rap1 controls telomere function and gene expression through binding to telomeric and extratelomeric sites. Nat Cell Biol.2010;12(8):768-780.

38. Frescas D, de Lange T. A TIN2 dyskeratosis congenita mutation causes telomerase-independent telomere shortening in mice. Genes Dev. 2014;28(2):153-166.

39. Jones $\mathrm{M}$, et al. Hematopoietic stem cells are acutely sensitive to Acd shelterin gene inactivation. JClin Invest. 2014;124(1):353-366.

40. Blanco R, Munoz P, Flores JM, Klatt P, Blasco MA. Telomerase abrogation dramatically accelerates TRF2-induced epithelial carcinogenesis. Genes Dev. 2007;21(2):206-220.

41. Munoz P, Blanco R, Flores JM, Blasco MA. XPF nuclease-dependent telomere loss and increased DNA damage in mice overexpressing TRF2 result in premature aging and cancer. Nat Genet. 2005;37(10):1063-1071.

42. Hockemeyer D, Palm W, Wang RC, Couto SS, de Lange T. Engineered telomere degradation models dyskeratosis congenita. Genes Dev. 2008;22(13):1773-1785.

43. $\mathrm{He} \mathrm{H}$, et al. Pot1b deletion and telomerase haploinsufficiency in mice initiate an ATR-dependent DNA damage response and elicit phenotypes resembling dyskeratosis congenita. Mol Cell Biol. 2009;29(1):229-240.

44. Wang Y, Shen MF, Chang S. Essential roles for Pot1b in HSC self-renewal and survival. Blood. 2011;118(23):6068-6077.

45. Beier F, Foronda M, Martinez P, Blasco MA. Conditional TRF1 knockout in the hematopoietic compartment leads to bone marrow failure and recapitulates clinical features of Dyskeratosis congenita. Blood. 2012;120(15):2990-3000.

46. Bilaud T, Brun C, Ancelin K, Koering CE, Laroche T, Gilson E. Telomeric localization of TRF2, a novel human telobox protein. Nat Genet. 1997;17(2):236-239.

47. Broccoli D, Smogorzewska A, Chong L, de Lange T. Human telomeres contain two distinct Myb-related proteins, TRF1 and TRF2. Nat Genet. 1997;17(2):231-235.

48. Chong L, et al. A human telomeric protein. Science.
1995;270(5242):1663-1667.

49. Zhong Z, Shiue L, Kaplan S, de Lange T. A mammalian factor that binds telomeric TTAGGG repeats in vitro. Mol Cell Biol. 1992;12(11):4834-4843.

50. van Steensel B, de Lange T. Control of telomere length by the human telomeric protein TRF1 Nature. 1997;385(6618):740-743.

51. Smogorzewska A, et al. Control of human telomere length by TRF1 and TRF2. Mol Cell Biol. 2000;20(5):1659-1668

52. Zhou XZ, Perrem K, Lu KP. Role of Pin2/TRF1 in telomere maintenance and cell cycle control. JCell Biochem. 2003;89(1):19-37.

53. Sfeir A, et al. Mammalian telomeres resemble fragile sites and require TRF1 for efficient replication. Cell. 2009;138(1):90-103.

54. Martinez P, et al. Increased telomere fragility and fusions resulting from TRF1 deficiency lead to degenerative pathologies and increased cancer in mice. Genes Dev. 2009;23(17):2060-2075.

55. Karlseder J, et al. Targeted deletion reveals an essential function for the telomere length regulator Trf1. Mol Cell Biol. 2003;23(18):6533-6541.

56. Celli GB, de Lange T. DNA processing is not required for ATM-mediated telomere damage response after TRF2 deletion. Nat Cell Biol. 2005;7(7):712-718.

57. Dimitrova N, de Lange T. Cell cycle-dependent role of MRN at dysfunctional telomeres: ATM signaling-dependent induction of nonhomologous end joining (NHEJ) in G1 and resectionmediated inhibition of NHEJ in G2. Mol Cell Biol. 2009;29(20):5552-5563.

58. Celli GB, Denchi EL, de Lange T. Ku70 stimulates fusion of dysfunctional telomeres yet protects chromosome ends from homologous recombination. Nat Cell Biol. 2006;8(8):885-890.

59. Konishi A, de Lange T. Cell cycle control of telomere protection and NHEJ revealed by a ts mutation in the DNA-binding domain of TRF2. Genes Dev. 2008;22(9):1221-1230.

60. Griffith JD, et al. Mammalian telomeres end in a large duplex loop. Cell. 1999;97(4):503-514.

61. Doksani Y, Wu JY, de Lange T, Zhuang X. Super-resolution fluorescence imaging of telomeres reveals TRF2-dependent T-loop formation. Cell. 2013;155(2):345-356.

62. Chen Y, et al. A conserved motif within RAP1 has diversified roles in telomere protection and regulation in different organisms. Nat Struct Mol Biol. 2011;18(2):213-221.

63. Li B, Oestreich S, de Lange T. Identification of human Rap1: implications for telomere evolution. Cell. 2000;101(5):471-483.

64. Sarthy J, Bae NS, Scrafford J, Baumann P. Human RAP1 inhibits non-homologous end joining at telomeres. $E M B O$ J. 2009;28(21):3390-3399.

65. Pardo B, Marcand S. Rap1 prevents telomere fusions by nonhomologous end joining. EMBO J. 2005;24(17):3117-3127.

66. Bae NS, Baumann P. A RAP1/TRF2 complex inhibits nonhomologous end-joining at human telomeric DNA ends. Mol Cell. 2007;26(3):323-334

67. Sfeir A, Kabir S, van Overbeek M, Celli GB, de Lange T. Loss of Rap1 induces telomere recombination in the absence of NHEJ or a DNA damage signal. Science. 2010;327(5973):1657-1661.
68. Martinez $\mathrm{P}$, et al. RAP1 protects from obesity through its extratelomeric role regulating gene expression. Cell Rep. 2013;3(6):2059-2074.

69. Yeung F, et al. Nontelomeric role for Rap1 in regulating metabolism and protecting against obesity. Cell Rep. 2013;3(6):1847-1856

70. Baumann P, Cech TR. Pot1, the putative telomere end-binding protein in fission yeast and humans. Science. 2001;292(5519):1171-1175.

71. Baumann P, Podell E, Cech TR. Human Pot1 (protection of telomeres) protein: cytolocalization, gene structure, and alternative splicing. Mol Cell Biol. 2002;22(22):8079-8087.

72. Lei M, Podell ER, Cech TR. Structure of human POT1 bound to telomeric single-stranded DNA provides a model for chromosome end-protection. Nat Struct Mol Biol. 2004;11(12):1223-1229.

73. Hockemeyer D, et al. Telomere protection by mammalian Pot1 requires interaction with Tpp1. Nat Struct Mol Biol. 2007;14(8):754-761.

74. Kibe T, Osawa GA, Keegan CE, de Lange T. Telomere protection by TPP1 is mediated by POT1a and POT1b. Mol Cell Biol. 2010;30(4):1059-1066.

75. Liu D, et al. PTOP interacts with POT1 and regulates its localization to telomeres. Nat Cell Biol. 2004;6(7):673-680.

76. Ye JZ, et al. POT1-interacting protein PIP1: a telomere length regulator that recruits POT1 to the TIN2/TRF1 complex. Genes Dev. 2004;18(14):1649-1654.

77. Palm W, Hockemeyer D, Kibe T, de Lange T. Functional dissection of human and mouse POT1 proteins. Mol Cell Biol. 2009;29(2):471-482.

78. Wu P, Takai H, de Lange T. Telomeric $3^{\prime}$ overhangs derive from resection by Exo1 and Apollo and fill-in by POT1b-associated CST. Cell. 2012;150(1):39-52.

79. Hockemeyer D, Daniels JP, Takai H, de Lange T. Recent expansion of the telomeric complex in rodents: two distinct POT1 proteins protect mouse telomeres. Cell. 2006;126(1):63-77.

80. Wu L, et al. Pot1 deficiency initiates DNA damage checkpoint activation and aberrant homologous recombination at telomeres. Cell. 2006;126(1):49-62.

81. Loayza D, De Lange T. POT1 as a terminal transducer of TRF1 telomere length control. Nature. 2003;423(6943):1013-1018.

82. Houghtaling BR, Cuttonaro L, Chang W, Smith S. A dynamic molecular link between the telomere length regulator TRF1 and the chromosome end protector TRF2. Curr Biol. 2004;14(18):1621-1631.

83. Else T, et al. Tpp1/Acd maintains genomic stability through a complex role in telomere protection. Chromosome Res. 2007;15(8):1001-1013.

84. Tejera AM, et al. TPP1 is required for TERT recruitment, telomere elongation during nuclear reprogramming, and normal skin development in mice. Dev Cell. 2010;18(5):775-789.

85. Wang F, et al. The POT1-TPP1 telomere complex is a telomerase processivity factor. Nature 2007;445(7127):506-510.

86. Latrick CM, Cech TR. POT1-TPP1 enhances telomerase processivity by slowing primer dissociation and aiding translocation. $E M B O J$ 2010;29(5):924-933.

87. Chen LY, Redon S, Lingner J. The human CST complex is a terminator of telomerase activity. 
Nature. 2012;488(7412):540-544.

88. Else T, et al. Genetic p53 deficiency partially rescues the adrenocortical dysplasia phenotype at the expense of increased tumorigenesis. Cancer Cell. 2009;15(6):465-476.

89. Ye JZ, et al. TIN2 binds TRF1 and TRF2 simultaneously and stabilizes the TRF2 complex on telomeres. J Biol Chem. 2004;279(45):47264-47271.

90. Takai KK, Kibe T, Donigian JR, Frescas D, de Lange $\mathrm{T}$. Telomere protection by TPP1/ POT1 requires tethering to TIN2. Mol Cell. 2011;44(4):647-659.

91. Chen LY, et al. Mitochondrial localization of telomeric protein TIN2 links telomere regulation to metabolic control. Mol Cell. 2012;47(6):839-850.

92. Chiang YJ, Kim SH, Tessarollo L, Campisi J, Hodes RJ. Telomere-associated protein TIN2 is essential for early embryonic development through a telomerase-independent pathway. Mol Cell Biol. 2004;24(15):6631-6634.

93. Walne AJ, Vulliamy T, Beswick R, Kirwan $\mathrm{M}$, Dokal I. TINF2 mutations result in very short telomeres: analysis of a large cohort of patients with dyskeratosis congenita and related bone marrow failure syndromes. Blood. 2008;112(9):3594-3600.

94. Savage SA, Giri N, Baerlocher GM, Orr N, Lansdorp PM, Alter BP. TINF2, a component of the shelterin telomere protection complex, is mutated in dyskeratosis congenita. Am J Hum Genet. 2008;82(2):501-509.

95. Sarper N, Zengin E, Kilic SC. A child with severe form of dyskeratosis congenita and TINF2 mutation of shelterin complex. Pediatr Blood Cancer. 2010;55(6):1185-1186.

96. Vulliamy T, Beswick R, Kirwan MJ, Hossain U, Walne AJ, Dokal I. Telomere length measurement can distinguish pathogenic from nonpathogenic variants in the shelterin component, TIN2. Clin Genet. 2012;81(1):76-81.

97. Vulliamy TJ, et al. Differences in disease severity but similar telomere lengths in genetic subgroups of patients with telomerase and shelterin mutations. PLoS One. 2011;6(9):e24383.

98. Goldman FD, et al. Characterization of primitive hematopoietic cells from patients with dyskeratosis congenita. Blood. 2008;111(9):4523-4531.

99. Townsley DM, Dumitriu B, Young NS. Bone marrow failure and the telomeropathies. Blood. 2014;124(18):2775-2783.

100.Balakumaran A, et al. Bone marrow skeletal stem/progenitor cell defects in dyskeratosis congenita and telomere biology disorders. Blood. 2015;125(5):793-802.

101. Mason PJ, Bessler M. The genetics of dyskeratosis congenita. Cancer Genet. 2011;204(12):635-645.

102.Savage SA. Human telomeres and telomere biology disorders. Prog Mol Biol Transl Sci. 2014;125:41-66.

103. Knight SW, et al. Unexplained aplastic anaemia, immunodeficiency, and cerebellar hypoplasia (Hoyeraal-Hreidarsson syndrome) due to mutations in the dyskeratosis congenita gene, DKC1. Br J Haematol. 1999;107(2):335-339.

104.Knight SW, et al. X-linked dyskeratosis congenita is predominantly caused by missense mutations in the DKC1 gene. Am J Hum Genet. 1999;65(1):50-58.
105. Walne AJ, et al. Genetic heterogeneity in autosomal recessive dyskeratosis congenita with one subtype due to mutations in the telomeraseassociated protein NOP10. Hum Mol Genet. 2007;16(13):1619-1629.

106.Vulliamy T, et al. Mutations in the telomerase component NHP2 cause the premature ageing syndrome dyskeratosis congenita. Proc Natl Acad Sci U S A. 2008;105(23):8073-8078.

107. Zhong F, et al. Disruption of telomerase trafficking by TCAB1 mutation causes dyskeratosis congenita. Genes Dev. 2011;25(1):11-16.

108. Ballew BJ, et al. Germline mutations of regulator of telomere elongation helicase 1 , RTEL1, in Dyskeratosis congenita. Hum Genet. 2013;132(4):473-480.

109. Walne AJ, Vulliamy T, Kirwan M, Plagnol V, Dokal I. Constitutional mutations in RTEL1 cause severe dyskeratosis congenita. Am J Hum Genet. 2013;92(3):448-453.

110.Le Guen T, et al. Human RTEL1 deficiency causes Hoyeraal-Hreidarsson syndrome with short telomeres and genome instability. Hum Mol Genet. 2013;22(16):3239-3249.

111. Cogan JD, et al. Rare variants in RTEL1 are associated with familial interstitial pneumonia. Am J Respir Crit Care Med. 2015;191(6):646-655.

112. Fedick AM, et al. Carrier screening of RTEL1 mutations in the Ashkenazi Jewish population. Clin Genet. 2015;88(2):177-181.

113. Anderson BH, et al. Mutations in CTC1, encoding conserved telomere maintenance component 1 , cause Coats plus. Nat Genet. 2012;44(3):338-342.

114. Polvi A, et al. Mutations in CTC1, encoding the CTS telomere maintenance complex component 1 , cause cerebroretinal microangiopathy with calcifications and cysts. Am J Hum Genet. 2012;90(3):540-549.

115. Keller RB, et al. CTC1 Mutations in a patient with dyskeratosis congenita. Pediatr Blood Cancer. 2012;59(2):311-314.

116. Stuart BD, et al. Exome sequencing links mutations in PARN and RTEL1 with familial pulmonary fibrosis and telomere shortening. Nat Genet. 2015;47(5):512-517.

117. Tummala H, et al. Poly(A)-specific ribonuclease deficiency impacts telomere biology and causes dyskeratosis congenita. JClin Invest. 2015;125(5):2151-2160.

118. Kocak H, et al. Hoyeraal-Hreidarsson syndrome caused by a germline mutation in the TEL patch of the telomere protein TPP1. Genes Dev. 2014;28(19):2090-2102.

119. Guo Y, et al. Inherited bone marrow failure associated with germline mutation of ACD, the gene encoding telomere protein TPP1. Blood. 2014;124(18):2767-2774.

120. Kirwan M, Dokal I. Dyskeratosis congenita: a genetic disorder of many faces. Clin Genet. 2008;73(2):103-112.

121. Armanios MY, et al. Telomerase mutations in families with idiopathic pulmonary fibrosis. N Engl JMed. 2007;356(13):1317-1326.

122. Alter BP, Rosenberg PS, Giri N, Baerlocher GM, Lansdorp PM, Savage SA. Telomere length is associated with disease severity and declines with age in dyskeratosis congenita. Haematologica. 2012;97(3):353-359.
123. Sasa GS, Ribes-Zamora A, Nelson ND, Bertuch AA. Three novel truncating TINF2 mutations causing severe dyskeratosis congenita in early childhood. Clin Genet. 2012;81(5):470-478.

124. Touzot F, et al. Heterogeneous telomere defects in patients with severe forms of dyskeratosis congenita. JAllergy Clin Immunol. 2012;129(2):473-482.

125. Glousker G, Touzot F, Revy P, Tzfati Y, Savage SA. Unraveling the pathogenesis of Hoyeraal-Hreidarsson syndrome, a complex telomere biology disorder. Br JHaematol. 2015;170(4):457-471.

126. Yang D, He Q, Kim H, Ma W, Songyang Z. TIN2 protein dyskeratosis congenita missense mutants are defective in association with telomerase. J Biol Chem. 2011;286(26):23022-23030.

127. Frank AK, Tran DC, Qu RW, Stohr BA, Segal DJ, Xu L. The Shelterin TIN2 Subunit Mediates Recruitment of Telomerase to Telomeres. PLoS Genet. 2015;11(7):e1005410.

128. Canudas S, et al. A role for heterochromatin protein 1gamma at human telomeres. Genes Dev. 2011;25(17):1807-1819.

129. Sexton AN, et al. Genetic and molecular identification of three human TPP1 functions in telomerase action: recruitment, activation, and homeostasis set point regulation. Genes Dev. 2014;28(17):1885-1899.

130. Poncet $\mathrm{D}$, et al. Changes in the expression of telomere maintenance genes suggest global telomere dysfunction in B-chronic lymphocytic leukemia. Blood.2008;111(4):2388-2391.

131. Augereau A, et al. Telomeric damage in early stage of chronic lymphocytic leukemia correlates with shelterin dysregulation. Blood. 2011;118(5):1316-1322.

132. Ramsay AJ, et al. POT1 mutations cause telomere dysfunction in chronic lymphocytic leukemia. Nat Genet. 2013;45(5):526-530.

133. Quesada V, et al. Exome sequencing identifies recurrent mutations of the splicing factor SF3B1 gene in chronic lymphocytic leukemia. Nat Genet. 2012;44(1):47-52.

134. Lin TT, et al. Telomere dysfunction and fusion during the progression of chronic lymphocytic leukemia: evidence for a telomere crisis. Blood. 2010;116(11):1899-1907.

135. Aoude LG, et al. Nonsense mutations in the shelterin complex genes ACD and TERF2IP in familial melanoma. J Natl Cancer Inst. 2015;107(2):dju408.

136. Robles-Espinoza CD, et al. POT1 loss-of-function variants predispose to familial melanoma. Nat Genet. 2014;46(5):478-481.

137. Shi J, et al. Rare missense variants in POT1 predispose to familial cutaneous malignant melanoma. Nat Genet. 2014;46(5):482-486.

138. Spinella JF, et al. A novel somatic mutation in $\mathrm{ACD}$ induces telomere lengthening and apoptosis resistance in leukemia cells. BMC Cancer. 2015;15:621.

139. Bainbridge MN, et al. Germline mutations in shelterin complex genes are associated with familial glioma. J Natl Cancer Inst. 2015;107(1):384.

140. Calvete O, et al. A mutation in the POT1 gene is responsible for cardiac angiosarcoma in TP53-negative Li-Fraumeni-like families. Nat Commun. 2015;6:8383. 